\title{
Implantação de Processo de Software na BL Informática - Um Caso de Sucesso
}

\author{
Analia Irigoyen Ferreiro Ferreira ${ }^{1}$, Roberta Cerqueira ${ }^{1}$, Ana Regina Rocha ${ }^{2}$, \\ Gleison Santos', Mariano Montoni², Sômulo Mafra², Sávio Figueiredo² \\ ${ }^{1}$ BL Informática Ltda. \\ Av. Visconde do Rio Branco 305/8 andar - Niterói - RJ - CEP: 24020-002 \\ ${ }^{2}$ COPPE/UFRJ - Universidade Federal do Rio de Janeiro \\ Caixa Postal 68511 - CEP 21945-970 - Rio de Janeiro, Brasil \\ \{analia, roberta.cerqueira\} ablnet.com \\ \{darocha, gleison, mmontoni, somulo, savio\}@cos.ufrj.br
}

\begin{abstract}
During 2003 and 2004, BL Informática was involved with the selection, preparation and deployment of a defined software process. The objective of this paper is to present in details the experience that resulted in the achievement of the ISO 9001:2000 by the organization. This work also presents some difficulties, success factors and lessons learned as the main results of the software process deployment.
\end{abstract}

Resumo. Durante os anos de 2003 e 2004, a BL Informática esteve envolvida com a escolha, preparação e implantação de um processo definido de software. $O$ objetivo deste relato é detalhar esta experiência que culminou com a obtenção da certificação ISO 9001:2000 pela empresa. Além disso, são apresentadas as dificuldades encontradas, fatores de sucesso e lições aprendidas como resultado da implantação do processo.

\section{Introdução}

Para manter o sucesso do negócio, organizações necessitam ser mais produtivas, aumentar a qualidade de seus produtos de software, diminuir o esforço e custos e lidar com o time-to-market para produtos comerciais [Pfleeger 2000]. Assim, a utilização de um processo de software de qualidade, mais do que uma metodologia torna-se então um aspecto fundamental para empresas que queiram permanecer no mercado [Rocha et al. 2001]. No contexto da indústria de software brasileira, um reflexo dessa crescente busca por utilização de processos de software de qualidade, pode ser observado por iniciativas como o projeto mps Br (Melhoria de Processo do Software Brasileiro) [Weber et al. 2004], que visa à melhoria de processos de software no contexto de pequenas e médias empresas brasileiras.

A BL Informática é uma empresa de desenvolvimento de software sediada em Niterói, no estado do Rio de Janeiro. Seu principal foco de negócio é desenvolvimento de software, integração, manutenção, implantação e fábrica de software. Desde sua criação, em 1987, a empresa propicia aos seus clientes uma melhoria significativa na administração dos seus negócios, através da melhor utilização da tecnologia da informação, da racionalização dos seus processos, do uso da opção tecnológica adequada e do aumento da qualidade das suas informações. Ao longo desses anos, 
auxiliando inúmeras organizações, a empresa acumulou conhecimentos nas diversas áreas de TI, investiu continuamente em treinamento e capacitou-se nas mais diversas tecnologias, tendo por objetivo principal a aplicabilidade, sob o prisma da produtividade, oportunidade e adequação às tendências do mercado.

Este artigo tem por objetivo relatar a experiência da BL Informática iniciada em 2003 na implantação de processos visando a aumentar a qualidade no desenvolvimento de software. A principal motivação da empresa nesta implantação foi a obtenção da Certificação ISO 9001:2000 [ISO 9001, 2000]. Um dos resultados esperados desde o princípio foi a redução de custos, uma vez que os produtos seriam produzidos com maior qualidade e, com isso, haveria a redução do re-trabalho.

De forma mais detalhada, neste artigo são apresentadas informações referentes às fases da implantação deste processo. A seção 2 relata como foi a escolha do processo e a preparação para a implantação. Na seção 3 é discutida a implantação do processo, destacando pontos chaves para realizá-la. A adaptação do processo para o Projeto Piloto, incluindo os obstáculos enfrentados e as decisões tomadas, é descrita na seção 4. Os resultados obtidos e as lições aprendidas com a implantação, e como isso refletiu na empresa, são apresentados na seção 5. Por fim, a seção 6 apresenta as considerações finais.

\section{Início}

O primeiro passo rumo à obtenção da certificação ISO 9001:2000, principal meta da empresa para o ano de 2004, foi a escolha dos processos de desenvolvimento e manutenção de software compatíveis com esta norma. Outro importante critério para a escolha dos processos era que, de alguma forma, eles já tivessem sido testados e fossem capazes de fornecer produtos com qualidade. Apesar de a escolha do processo não ter sido fácil, foi rápida.

A consultoria da COPPE/UFRJ foi solicitada para ajudar na confecção destes processos de software. Por coincidência, no mesmo ano de 2003, foi criado o Projeto QUALISOFT [Santos et al. 2005], idealizado pela RioSoft (organização nãogovernamental que integra o Programa Softex - Sociedade para Promoção da Excelência do Software Brasileiro) com a parceria da COPPE/UFRJ e subsídio do SEBRAE/RJ. O objetivo do Projeto QUALISOFT é a melhoria e aumento da produtividade das empresas, com a adoção das melhores práticas da Engenharia de Software, concretizada na implementação de um processo padrão de desenvolvimento de software com qualidade, baseado na norma ISO/IEC 12207 [ISO/IEC 2000]. A BL Informática formalizou a participação na primeira fase do programa e, com isto, a escolha do processo foi realizada.

O Projeto QUALISOFT englobou as seguintes fases: (i) treinamento em Engenharia de Software para auxiliar a utilização do processo oferecido; (ii) escolha pela organização de um Projeto Piloto, com duração prevista de 3 meses, para a utilização do processo; (iii) adaptação do processo QUALISOFT oferecido à cultura de cada empresa; (iv) utilização do Ambiente TABA [Oliveira et al. 2004], cujo objetivo é apoiar a utilização do processo; (v) consultoria da COPPE/UFRJ para auxílio à implantação do processo de software e das ferramentas de apoio. 
Treinamento - Todos os profissionais da BL que participariam da implantação do processo fizeram o treinamento, ministrado pela equipe da COPPE, nas disciplinas: Engenharia de Software, Engenharia de Requisitos, Qualidade de Processos, Gerência de Configuração, Gerência de Projetos e Qualidade de Produto. O treinamento foi realizado nas dependências da RioSoft, teve a duração de 32 horas e foi compartilhado por todas as empresas participantes do projeto QUALISOFT.

Projeto Piloto - Como forma de disseminar a utilização do processo, foi escolhido o desenvolvimento de um software médio, para modalidade ASP, uma vez que a previsão de conclusão do projeto era de 6 meses. O objetivo principal deste projeto, denominado BLFactoring, era a construção de um software para gerenciar e controlar descontos de títulos.

A Estação TABA - A Estação TABA [Oliveira et al. 2004] é um Meta-Ambiente de Desenvolvimento de Software criado para apoiar a atividades individuais relacionadas à execução e gerência de projetos, melhoria da qualidade de produtos de software e aumento da produtividade, proporcionando os meios para que os engenheiros de software controlem o projeto e mensurem a evolução das atividades baseada em informações adquiridas através do desenvolvimento. A Estação TABA também provê a infra-estrutura para o desenvolvimento de ferramentas integradas para apoiar a execução dos processos de software. Mais ainda, esta infra-estrutura contém um repositório de informações sobre o projeto de software adquirido através de seu ciclo de vida.

Dois tipos de ambientes gerados pela Estação TABA foram disponibilizados para empresas participantes do projeto QUALISOFT: (i) Ambiente Configurado: responsável por informações padronizadas da organização. Neste ambiente é possível registrar características específicas da organização, como o modelo de um artefato, as métricas definidas pela empresa, estrutura e mapa de conhecimento da organização e a aquisição e disseminação do conhecimento organizacional. Além disso, permite a instanciação de ambiente de desenvolvimento de software específico para um projeto. (ii) Ambiente Instanciado: responsável por informações padronizadas de um projeto específico. É neste ambiente que acontece a execução e controle de um projeto durante todo o seu ciclo de vida apoiado por ferramentas disponíveis no ambiente. Essas ferramentas incluem planejamento de tempo, custo, riscos, recursos humanos, gerência de configuração, medição e análise, identificação dos requisitos de qualidade do produto, documentação, gerência de requisitos etc.

\section{Implantação de um novo processo}

Embora houvesse uma cultura de utilização de metodologias por parte da empresa, a simples proposta de mudança para um outro processo foi alvo de muitas críticas. Esta etapa consumiu uma grande quantidade de tempo. O processo de software do Projeto QUALISOFT exigia a aplicação de conceitos de Engenharia de Software e Qualidade de Software, cujo conteúdo gerou confusão e, num primeiro momento, até a sua não aceitação por desconhecimento da área. Foram necessárias muitas reuniões de conscientização, ressaltando a necessidade do comprometimento dos colaboradores e o quanto a certificação ISO era importante para a empresa. Houve uma certa dificuldade em convencer que o processo de software oferecido era eficaz e produtivo. Isso ocorreu 
porque o processo tinha um número muito maior de artefatos do que a metodologia utilizada anteriormente, estes artefatos tinham roteiros, não familiares para a equipe, que deveriam ser seguidos e o prazo de implantação era curto (6 meses).

Numa tentativa de minimizar o impacto durante a implantação do processo, a princípio foi decidido a utilização somente do processo proposto pelo Projeto QUALISOFT, deixando a utilização da Estação TABA para uma outra fase. O receio da equipe naquele momento era que a utilização da Estação TABA fosse aumentar a curva de aprendizado e, assim, aumentar o tempo de obtenção da certificação.

No fim desta fase, ainda existiam pessoas da equipe de implantação que não aceitavam o processo a ser utilizado e que acreditavam que muitas customizações ainda seriam necessárias. Neste momento, a alta direção da empresa teve um papel fundamental ao incentivar e apoiar a continuação da implantação do processo.

\section{Adaptação do Processo para o Projeto Piloto}

A fase de adaptação do processo foi a mais trabalhosa para a equipe líder da implantação. Foi preciso agendar um maior número de pontos de controle para verificar se artefatos tinham sido criados e se os roteiros tinham sido seguidos. Estas reuniões também tinham como objetivo esclarecer sobre termos e boas práticas de Engenharia de Software. Durante esta fase foram feitas muitas adaptações de roteiros, artefatos gerados e inclusão de atividades. Também houve a integração deste processo de software com os outros processos da empresa: Gerenciamento de Propostas, Gerenciamento de Contratos, Treinamento Organizacional, Assistência Técnica e Garantia. Foi decidido, como forma de documentação e objetivando o aprendizado organizacional, que todas as alterações no processo padrão seriam justificadas e as lições aprendidas em cada etapa seriam registradas.

Inicialmente, o processo foi utilizado sem a ajuda de ferramenta específica para gerenciamento durante toda a fase de Análise. Foram criados artefatos, estrutura de diretórios, política de segurança e outros controles necessários para obter uma infraestrutura que permitisse, dentre outras coisas, armazenar os modelos de documentos do processo padrão e dos projetos específicos, efetuar o controle de versões, garantir que todos os desenvolvedores utilizassem os modelos de documentos corretos e realizar o planejamento da documentação.

A dificuldade na estruturação destes mecanismos de controle apontou para a necessidade de um apoio ferramental para auxiliar na utilização do processo e, também, no planejamento, controle e execução do projeto. Devido à percepção desta necessidade, a utilização da Estação TABA voltou a ser considerada. A partir deste momento, os recursos do ambiente Configurado começaram a serem utilizados em sua totalidade, tais como: registro das lições aprendidas, dúvidas e justificativas de alterações no processo padrão. Já o Ambiente Instanciado (que apóia a execução e controle do projeto) era utilizado somente para registrar o início e o fim das atividades. À medida que a utilização do processo avançava, as ferramentas adicionais da Estação TABA começaram a ser utilizadas gradativamente, apoiando cada etapa da execução do processo.

Em paralelo, a adaptação do processo à cultura da empresa continuou sendo realizada, mas sem descaracterizar o processo original do Projeto QUALISOFT. Esta 
adaptação incluiu: (i) disponibilização de equipe dedicada exclusivamente à implantação do processo; (ii) divulgação interna do processo indicando boas práticas definidas; (iii) realização de mini-treinamentos pela própria equipe de implantação para ajudar no entendimento de questões de Engenharia de Software; (iv) definição de um maior número de pontos de controle durante as fases do processo com a realização de reuniões semanais para tirar dúvidas sobre o que estava impedindo a execução do processo ou tinha impacto na produtividade em uma determinada atividade; (v) realização, durante a fase de codificação, de inspeção em $100 \%$ dos artefatos gerados até que o número de erros fosse diminuído significativamente; (vi) ajustes das definições de padrões de desenvolvimento, por exemplo: nomenclatura, dicionarização, templates, boas práticas de programação, otimização de SQL etc; (vii) discussão dos erros mais comuns sendo cometidos e disponibilidade para ajudar no entendimento ou realizar mini-cursos para suprir as necessidades de treinamento; (viii) incentivo da equipe através de relatórios contendo indicadores de melhoria alcançados; (ix) realização de reuniões freqüentes de 1 hora cada, onde eram discutidos o andamento do projeto e sua aderência ao processo, instruções sobre como elaborar artefatos e seguir os modelos de documentos, discussão de dúvidas e agendamento de mini-treinamentos para solucioná-las, discussão das causas e soluções dos maiores erros encontrados no relatório de inspeção.

Não só o processo de software sofreu adaptações à cultura da empresa. A equipe de desenvolvimento também teve que se adaptar ao processo, por exemplo, modificando a forma de realização de algumas atividades previstas no processo e que já existiam na metodologia de desenvolvimento anterior.

Durante a fase de implantação, quando o projeto piloto já estava na fase de construção, houve uma alteração no cronograma do projeto piloto inicial. Foi decidido, então, começar um novo projeto piloto, com duração de 1 mês, para que todo o processo fosse executado. O segundo projeto piloto, considerado de pequeno porte, tinha como objetivo a constução de um Classificador de Impressões, denominado Agente BL. No fim da fase de implantação do processo, o segundo projeto piloto, AgenteBL, já havia sido concluído, e o projeto piloto inicial, BLFactoring, estava ainda na atividade de construção. Em ambos os casos os clientes estavam satisfeitos com os projetos.

Apesar do projeto BLFactoring não ter terminado no prazo, sua execução foi considerada um sucesso. Os clientes acompanharam de perto todo o processo, tomando conhecimento dos artefatos e das não-conformidades detectadas e realizando avaliações ao final de cada atividade. As alterações solicitadas foram identificadas como evolutivas e o prazo e o custo do projeto foram renegociados. O impacto real das alterações foi facilmente mensurado através da rastreabilidade dos requisitos, cuja identificação é prevista no processo do projeto QUALISOFT.

Ao final desta primeira etapa, já com o processo estabilizado, um ano havia se passado. O tempo gasto não foi o mesmo planejado inicialmente, mas bons resultados, tanto técnicos quanto de relacionamentos interpessoais, já tinham sido identificados.

\section{Resultados e Lições Aprendidas}

Os frutos da implantação podem ser comprovados em relatórios dos Indicadores x Metas estabelecidas e também através da obtenção da Certificação ISO 9001:2000 no final de 2004. Numa análise inicial dos relatórios de Indicadores x Metas poder-se-ia chegar 
numa conclusão que a equipe está levando mais tempo para realizar algumas das tarefas. $\mathrm{Na}$ verdade, apesar do tempo gasto ser maior não significa que o custo total final do projeto tenha aumentado, pois o re-trabalho diminuiu e, o mais importante, o número de erros na avaliação do cliente foi reduzido drasticamente. Assim, a possibilidade de atrasos no cronograma ou aumento do custo não repassado ao cliente foi diminuída. Esses fatores são considerados muito importantes pela equipe de desenvolvimento.

As Figuras 1 e 2 mostram o gráfico de produtividade de 2 projetos da BL.

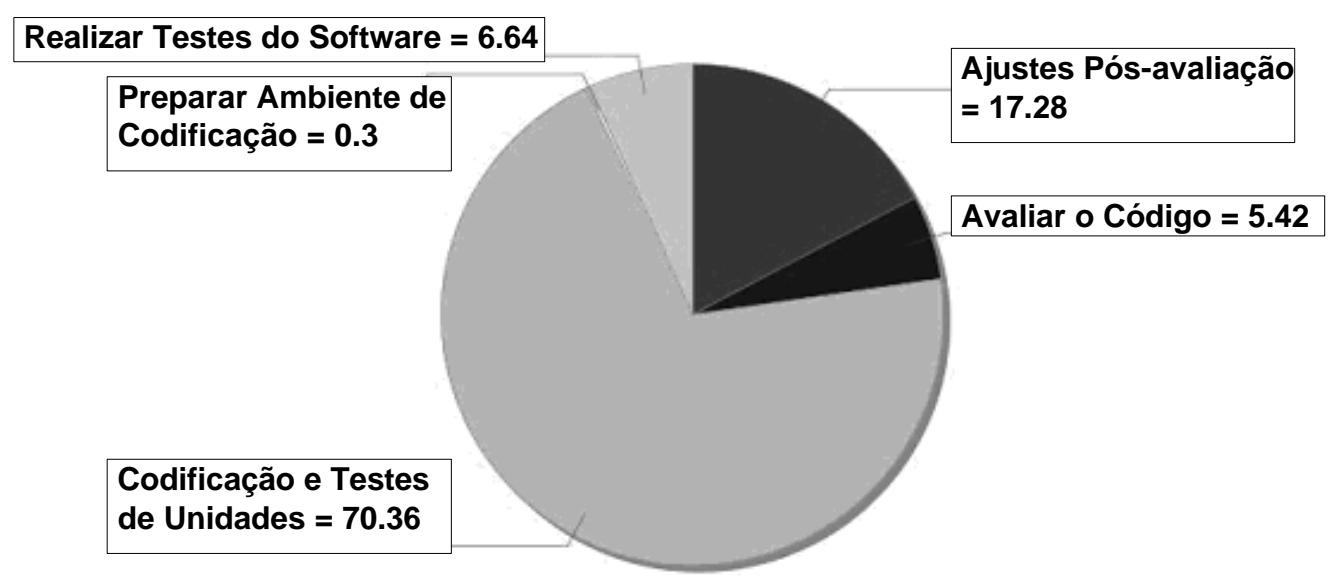

Figura 1 - Gráfico de produtividade no projeto BLFactoring

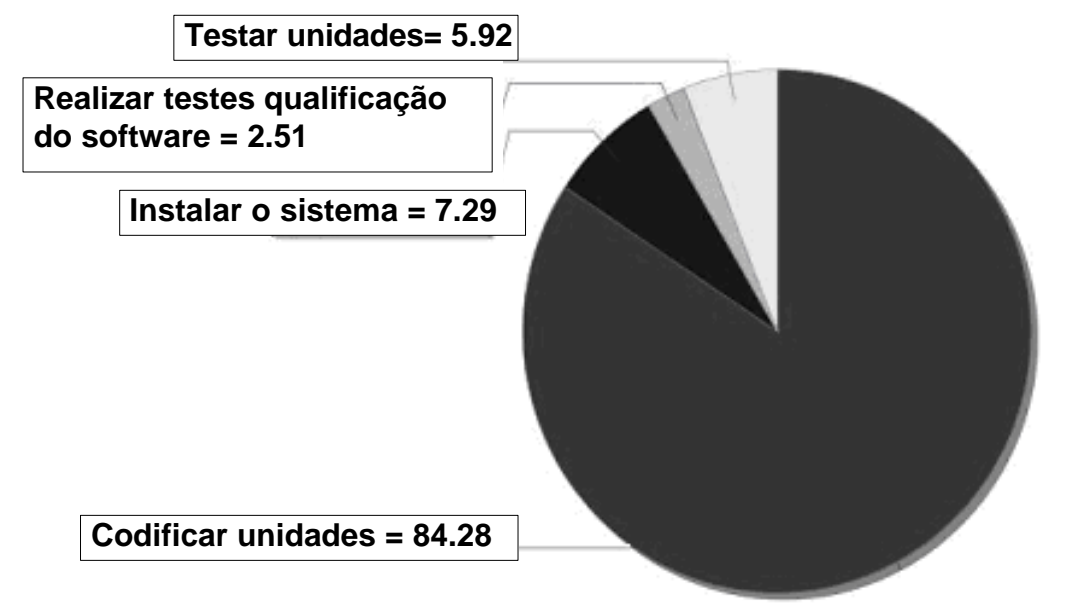

Figura 2 - Gráfico de produtividade no projeto AgenteBL

No projeto BLFactoring (primeiro projeto piloto iniciado) o tempo gasto em ajustes após a avaliação do cliente foi cerca de $25 \%$ do tempo gasto na codifição do software. No projeto AgenteBL, iniciado após o projeto piloto e, portanto, com o processo mais maduro na organização, o tempo gasto em ajustes após a avaliação do cliente foi praticamente nenhum. Isso foi possível devido, em grande parte, às freqüentes avaliações internas e com o cliente previstas no processo do QUALISOFT.

Implantar um processo de software com qualidade exigiu mais do que o esperado a princípio, mas em compensação trouxe muito mais benefícios que os planejados inicialmente. Como principais benefícios, pode-se destacar: (i) redução da quantidade de 


\section{Simpósio Brasileiro de Qualidade de Software}

re-trabalho; (ii) maior qualidade dos artefatos gerados; (iii) melhor entendimento de Engenharia de Software através da qualificação dos colaboradores; (iv) objetivos bem claros e definidos sobre como a empresa trabalha e qual a qualidade e comprometimento esperados de seus colaboradores; (v) manutenção do conhecimento na empresa criando certa independência da equipe do projeto. As pessoas são importantes, mas o cliente não perderá a qualidade no atendimento dos seus requisitos se um membro da equipe tiver sido desalocado do projeto por algum motivo.

Dentre os fatores de sucesso para a implantação do processo, a equipe de implantação identificou que: (i) a alta direção estava comprometida e providenciou todo o aporte necessário, dentre os quais um consultor, especialista em implantar processos de software, acompanhando toda a fase de implantação; (ii) a utilização do processo facilita o desenvolvimento, pois não é preciso ficar pensando o que fazer, como fazer, quando fazer: tudo está escrito e formalizado; (iii) as reuniões freqüentes da equipe tiveram um papel fundamental na motivação dos participantes dos projetos piloto e demais projetos utlizando o processo QUALISOFT; (iv) a atenção, tempo e criatividade da equipe de desenvolvimento foram direcionados ao negócio do cliente, fazendo com que ele ficasse imensamente satisfeito; (v) a avaliação post mortem realizada ao final de cada projeto é um momento em que melhorias podem ser apontadas e, caso seja necessário, mudanças no processo sejam sugeridas. Com isso, podemos constatar que o processo não é estático, é saudavelmente dinâmico, como a tecnologia que nos cerca.

Além disso, algumas particularidades com relação à equipe e à empresa, fizeram com que fosse possível atingir mais rapidamente a maturidade na utilização do processo de desenvolvimento de software, a saber: (i) a empresa já possuía cultura em metodologia de desenvolvimento; (ii) a empresa já possuía padrões de desenvolvimento bem definidos; (iii) a equipe que liderou a implantação estava altamente comprometida e consciente dos benefícios de uma implantação de processo.

Outro fator decisivo para o sucesso da iniciativa foi possuir o apoio tecnológico de uma ferramenta como a Estação TABA. A sua utilização facilita e reduz significativamente o trabalho repetitivo durante a instanciação de um processo (criação de artefatos, roteiros, definição de ferramentas), planejamento (planejamento de: tempo, custo, documentos, recursos humanos, gerência de configuração, riscos, medição e análise, estrutura organizacional), controle (avaliações de qualidade, gerência de requisitos, monitoração dos itens planejados e gerenciamento dos planos de ação) e execução do projeto (registro das métricas definidas no plano de medição, acompanhamento das atividades realizadas e anexação dos artefatos criados e consumidos em cada atividade). Mais ainda, a Estação TABA possui uma abordagem integrada em todas as ferramentas para gerência de conhecimento contendo um repositório de lições aprendidas e registro de dúvidas, que serve como repositório de informações históricas para momentos críticos de tomada de decisão.

\section{Considerações Finais}

Este artigo apresentou a experiência da BL Informática na implantação de um processo de software dentro do contexto do projeto QUALISOFT que culminou com a obtenção da certificação ISO 9001:2000 pela empresa. A maior constatação de que o processo facilita o trabalho foi quando, por decisão da própria equipe, o processo começou a ser 
utilizado também em sistemas internos, que não estavam incluídos no escopo inicial.

Com a experiência obtida durante a fase anterior e a adição de novos controles, documentos e instruções técnicas, foi possível iniciar a Fábrica de Construção de Software seguindo o mesmo processo de software padrão definido na fase inicial. Foi decidido que a Fábrica de Software seria responsável pela execução das atividades do processo de software que englobam desde a etapa de Construção até a Homologação Interna. A Fábrica de Software está produzindo artefatos oficialmente desde setembro de 2004. E mais, além dos 2 projetos citados na seção anterior, há 2 outros projetos de manutenção em andamento seguindo o processo do QUALISOFT. O processo padrão já é mais natural e já está fazendo parte da cultura da empresa; ou seja, foi institucionalizado. A fase atualmente em curso é a melhoria contínua do processo.

O esforço da BL Informática não termina com os resultados obtidos até agora. Para o segundo semestre do ano de 2005 está planejada a avaliação mps Br nível $\mathrm{F}$ [Weber et al. 2004] no contexto das avaliações piloto e a avaliação CMMI nível 3 [Chrissis et al. 2003]. Estas metas foram consideradas possíveis de serem atingidas pela consultoria devido ao estágio atual dos processos na empresa.

\section{Agradecimentos}

Os autores agradecem ao Sebrae pelo apoio financeiro ao projeto QUALISOFT, a Benito Diaz Paret, coordenador da Riosoft, a Márcio Pecegueiro Amaral, coordenador do projeto QUALISOFT, à Diretoria da BL Informática, à equipe que participou na implantação do processo na BL e à equipe de desenvolvimento do Projeto TABA.

\section{Referências}

Chrissis, M. B., Konrad, M, Shrum, S., (2003), "CMMI: Guidelines for Process Integration and Product Improvement", Addison-Wesley.

ISO/IEC 12207:2000, (2000), "Information technology - software process life cycle".

ISO 9001:2000, (2000), "Quality management systems - Requirements".

Oliveira, K, Zlot, F., Rocha, A. R., Travassos, G., Galotta, C., Menezes, C., (2004) "Domain Oriented Software Development Environment", Journal of Systems and Software, vol 72/2 pp 145-161.

Pfleeger, S. L., (2001), "Software Engineering: theory and practice", 2nd edition, Prentice-Hall, Inc.

Rocha, A. R. C., Maldonado, J. C., Weber, K. C., et al., (2001), "Qualidade de Software: Teoria e Prática”, São Paulo: Prentice Hall.

Santos, G., Montoni, M., Rocha, A.R., Figueiredo, S., Mafra, S., Albuquerque, A., Paret, B., Amaral, M., (2005), "Using a Software Development Environment with Knowledge Management to Support Deploying Software Processes in Small and Medium Size Companies", 7th Int. Workshop on Learning Software Organizations (LSO'2005), Kaiserslautern, Alemanha, April.

Weber, C. K., Rocha, A. R; Alves, A, et al. (2004), "Modelo de Referência para Melhoria de Processo de Software: uma abordagem brasileira". XXX Conferencia Latino-americana de Informática, Arequipa - Peru. 